\title{
The Effect of Curcumin Supplementation and Aerobic Training on Anthropometric Indices, Serum Lipid Profiles, C-Reactive Protein and Insulin Resistance in Overweight Women: A Randomized, Double-Blind, Placebo-Controlled Trial
}

\author{
Sepideh Dolati ${ }^{1}$, Khoosheh Namiranian ${ }^{2}$, Reyhaneh Amerian ${ }^{3}$, Samane Mansouri ${ }^{4}$, Sajad Arshadi ${ }^{5}$, Mohammad Ali Azarbayjani ${ }^{5, *}$ \\ 'Department of Nutrition, Ministry of Health, Tehran; ${ }^{2}$ Department of Health Tehran University of Medical Sciences, Tehran; ${ }^{3}$ Department of Exercise Physiology, \\ Faculty of Physical Education, Islamic Azad University of Tehran, Tehran; ${ }^{4}$ Nutrition Group, Abadan School of Medical Sciences, Abadan; ${ }^{5}$ Department of Exercise \\ Physiology, Faculty of Physical Education and Sports Science, Islamic Azad University, Central Tehran Branch, Tehran, Iran
}

Background: This study aimed to investigate the effects of curcumin supplementation alone and in combination with aerobic training on body composition, glycemic variables, serum levels of C-reactive protein (CRP) and lipid profiles in overweight women.

Methods: In this randomized, double-blind, placebo-controlled trial, 40 healthy sedentary overweight females (aged 30-45 years with body mass index [BMl] of $25-30 \mathrm{~kg} / \mathrm{m}^{2}$ ) were randomly assigned to four groups of 10 subjects each: curcumin supplementation (Cur), placebo (Pla), placebo+aerobic training (Pla+Tra), curcumin supplementation+aerobic training (Cur+Tra). Curcumin or placebo capsules ( $500 \mathrm{mg} /$ day) were administered for 8 weeks.

Results: During the intervention, Cur group showed significant reductions in weight, BMI and fasting blood glucose level; Cur+Tra group decreased waist circumference, waist-to-hip ratio, homeostatic model assessment for insulin resistance, serum insulin level, low-density lipoprotein to high-density lipoprotein ratio, and total cholesterol to high-density lipoprotein ratio and increased serum high-density lipoprotein cholesterol. The between-groups analysis indicated increased high-density lipoprotein cholesterol in the Cur and Cur+Tra groups compared to the Pla group. The estimated marginal means of serum CRP were significantly higher in Pla+Tra group than in the Cur and Cur+Tra groups.

Conclusion: These findings suggest that the combination of curcumin supplementation with aerobic training more effectively improves glycemic and lipidemic statuses than curcumin supplementation or aerobic training alone.

Key words: Curcumin, Exercise, Overweight, C-reactive protein, Lipids, Insulin resistance
Received October 8, 2019

Reviewed November 7, 2019

Accepted January 27, 2020

*Corresponding author

Mohammad Ali Azarbayjani

https://orcid.org/0000-0002-3502-7487

Department of Exercise Physiology, Faculty of Physical Education and Sports Science, Islamic Azad University, Central Tehran Branch, Army Boulevard, Shahid Sohani St., Sohank Square, Tehran 1986755881, Iran

Tel: +98-9106759828

Fax: +98-81454357

E-mail: M_azarbayjani@iauctb.ac.ir

The first two authors contributed equally to this study.

\section{INTRODUCTION}

Currently, overweight and obesity are problems around the world, especially for women. ${ }^{1,2}$ In developing countries, it seems that the prevalence of obesity among women is more than that among men. ${ }^{3}$ This condition has an important effect on health impairment, female reproduction ${ }^{4}$ and reduced quality of life. ${ }^{5}$ Overweight and obesity are associated with diseases such as cardiovascular disease, 
diabetes, various cancers, nervous system disorders and mortality. ${ }^{6,7}$ Studies have shown that overweight and obesity are associated with insulin resistance index. It seems these factors cause many complications, metabolic problems and some diseases. ${ }^{3,8-10}$

Recent studies have focused on a variety of strategies such as physical activity, herbal therapy, dietary supplements, and pharmacological intervention for weight management as well as the effects on insulin resistance in overweight and obese patients..$^{8-11}$ Various studies have indicated that physical activity is an important environmental factor associated with body weight regulation. Increasing physical activity has become an important aspect of nonpharmacological strategies to control obesity and blood glucose, as well as to prevent impaired lipid profile advancement and glucose intolerance progression. ${ }^{12}$ Furthermore, it has been reported that curcumin may have protective effects against obesity and the risk of chronic diseases such as cardiovascular diseases, diabetes, and several cancers. ${ }^{13,14}$

Curcumin is a bioactive polyphenol found in turmeric rhizomes and is used in traditional Chinese medicine. ${ }^{15,16}$ There is evidence that in people with metabolic syndrome, curcumin can decrease body mass index (BMI), weight, waist circumference (WC) and leptin concentrations. ${ }^{17}$ In individuals with metabolic syndrome, the development of inflammatory mediators is the basis of biological disorders. Curcumin plays an inhibitory role in the development of inflammation in these patients. Curcumin decreased the release of cytokines and the permeability of the M1 family of macrophages and improved insulin sensitivity by activating SIRT1 in adipose tissue. ${ }^{18}$ Many studies have shown that curcumin has antiinflammatory, anti-infectious, anti-oxidant, anti-arthritic, cardioprotective, anti-carcinogenic and anti-anxiety effects in obese individuals. ${ }^{19,20}$ It may help to improve conditions such as asthma, allergies, bronchial hyperactivity, sinusitis, anorexia, cough and hepatic disease and to inhibit cancer progression. ${ }^{21-23}$ The anti-inflammatory property of curcumin appears to be mediated through the inhibition of the induction of cyclooxygenase-2 (COX-2), lipoxygenase, inducible nitric oxide synthase, and the production of cytokines such as tumor necrosis factor and C-reactive protein (CRP) ${ }^{24}$

Most studies have explored the effects of training or curcumin supplementation separately on anthropometric indices, lipid profiles, CRP and insulin resistance. ${ }^{25,26}$ However, few studies have been done on the simultaneous effects of curcumin and physical activity on these factors. Therefore, we investigated the effects of curcumin supplement intake alone and in combination with aerobic training on body composition, glycemic variables, lipid profiles, and CRP in overweight women.

\section{METHODS}

\section{Subjects}

Forty healthy, sedentary (less than 30 minutes a day of moderate intensity physical activity for 3 days a week $\left.{ }^{27,28}\right)$, overweight (24.9 $<$ BMI $<29.9 \mathrm{~kg} / \mathrm{m}^{2}$ ) women aged 30-45 years were recruited from those referred to the comprehensive health center or health center located in the Yaftabad district of the Tehran University of Medical Sciences. In the random or accidental method used in this study, the choosing and investigation of subjects were completely random, such that each subject had the same chance of being chosen. In this method, a simple randomizing model like the drawing of lots was employed. In this case, the subjects were assigned to the control or intervention groups according to the lots results. The Fleiss equation for sample volume estimation was used in this survey in order to approximate the volume of samples, in which the power of the experiment, the alpha of the equation, and the average alteration were $0.8,0.05$, and 5 , respectively. According to the conducted estimation, the sample size was calculated to be 8.81 . To be more conservative, among the overweight volunteer women, 10 people were chosen for the experimental group.

Exclusion criteria for our study were allergy to curcumin, physical activity or aerobic exercises of any form and type in the past 6 months, pregnancy, underlying or infectious disease, following a special diet, intake of dietary supplements and herbal tea, smoking or other tobacco and hookah consumption, a history of high blood lipids without medication, cardiac failure, taking anti-inflammatory drugs, severe endocrine or metabolic disorders or hospitalization for any reason.

\section{Study design}

This study was designed as a double-blind, randomized, placebocontrolled clinical trial and performed between January 2018 and March 2018. A flowchart of the study protocol is shown in Fig. 1. 


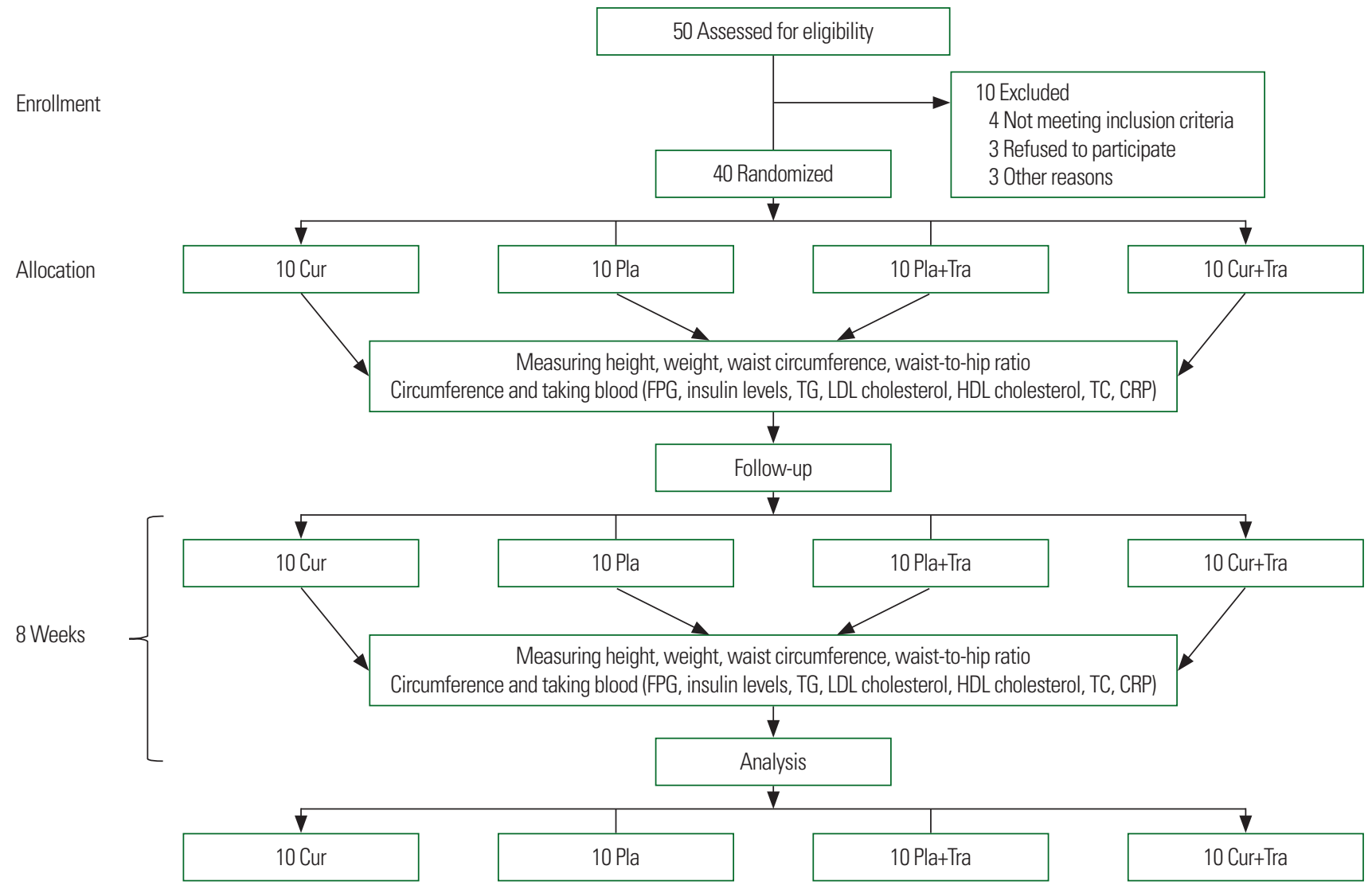

Figure 1. A flowchart of the study protocol. Cur, curcumin supplement; Pla, placebo; Pla+Tra, placebo+aerobic training; Cur+Tra, curcumin supplement+aerobic training; FPG, fasting plasma glucose; TG, triglyceride; LDL, low-density lipoprotein; HDL, high-density lipoprotein; TC, total cholesterol; CRP, C-reactive protein.

The subjects $(n=40)$ were randomly allocated using a list prepared with a random number generator into four groups of 10: curcumin supplementation (Cur), placebo (Pla), placebo+aerobic training (Pla+Tra), curcumin supplementation+aerobic training (Cur+Tra) (Fig. 1). For 8 weeks, the Cur and Tra groups ingested two curcumin capsules per day (500 mg/day), and the Pla and Pla+Tra groups received a placebo every day.

The project was approved by the Ethical Committee at the Azad University, Tehran Branch, registered in the Iranian Registry of Clinical Trials (No. IR.SSRI.REC.1397.382), and written informed consent was obtained from all participants.

\section{Supplementation}

The turmeric powder was made by a reputable manufacturer, Golha Company, under supervision according to the standard processing procedure of pharmaceutical materials (Analysis Center of the Institute of Medicinal Plants). Then, 5,000 $\mathrm{g}$ of the powder was poured into $5,000 \mathrm{~mL}$ of alcohol at $80^{\circ} \mathrm{C}$. The percolation method was used for the extraction by alcohol. After 24 hours, the contents were smoothed using a Buchner funnel and ordinary filter paper. The extraction efficiency was $10 \%$. The powder was dispersed in a suitable solvent ( $80 \%$ ethanol) to prepare the drug form. Next, breadcrumbs were used as an expander and filler to achieve proper fluidity. Based on previous clinical studies, an effective dose of 250 $\mathrm{mg}$ of the ethanolic extract is estimated to be $80 \%$. Total curcuminoids have been estimated at $75 \%$ to $85 \%$ in previous studies. ${ }^{29}$ Therefore, 250-mg capsules (00 short stays; Iran Gelatin Company, Tehran, Iran) were prepared with a semiautomatic encapsulating machine. The curcumin extract concentration was analyzed by the high-performance liquid chromatography method ( $85 \%$ curcuminoids, including curcumin, demethoxycurcumin, and bisdemethoxycurcumin). Each capsule contained $250 \mathrm{mg}$ of product. Subjects in the Cur and Cur+Tra groups were provided with two capsules of curcumin per day. Likewise, the Pla and Pla+Tra groups re- 
ceived two placebo capsules per day, which were similar in shape and color and made of an equivalent dose of starch. The study duration was 8 weeks, and all subjects were instructed not to alter their dietary habits during the intervention period.

\section{Aerobic training}

Subjects in the Cur+Tra and Pla+Tra groups underwent aerobic training ( 3 days per week for 8 weeks) in a gym. The aerobic capacity of each subject was examined by a 6-minute Rockport test. The maximum heart rate for each person was estimated as equal to $208-0.7 \times($ age $) .{ }^{30}$ In the training session, subjects were first warmed up for 6 minutes with stretching exercises and then ran continuously on the treadmill at a speed of $7 \mathrm{~km} / \mathrm{hr}$, at a heart rate of $50 \%$ to $80 \%$ of maximum, for 20 minutes. Their heart rate was monitored by a polarimeter (Polar, Kempele, Finland; within a 5\% error rate of a heartbeat) during running to maintain the specified intensity. This intensity was individualized. Every person had a certain intensity. The younger subjects ran with higher intensity and the older ones ran with lower intensity. In each of the next sessions, $90 \mathrm{sec}-$ onds were added to the running time. The training intensity was controlled using a belt heart rate sensor (Polar beat, Kempele, Finland). Subjects in the Cur and Pla groups were instructed not to change their level of physical activity.

\section{Anthropometric measurements}

Anthropometric indexes were measured by one observer (SD). Body weight was measured by electronic digital scales, which were accurate to $0.1 \mathrm{~kg}$ with subjects in light clothing and no shoes.
Height was measured in the standing position to the nearest $0.1 \mathrm{~cm}$ using a flexible stadiometer. BMI was calculated by dividing body weight $(\mathrm{kg})$ by height squared $(\mathrm{m})$. The waist and hip circumferences ( $\mathrm{HC}$; to the nearest $0.5 \mathrm{~cm}$ ) were collected in the standing position according to standardized methodologies. ${ }^{31}$ The WC was measured from the front at the narrowest point between the rib cage and iliac crest after full expiration, while the $\mathrm{HC}$ was measured from the side at the maximal extension of the buttocks using an ordinary measuring tape. Waist-to-hip ratio (WHR) was calculated based on the WC $(\mathrm{cm})$ divided by $\mathrm{HC}(\mathrm{cm})$.

\section{Laboratory tests}

Ten milliliters of blood were obtained from the left vein in the sitting position from each patient in the early morning after overnight fasting. Then, samples were centrifuged and analyzed. Fasting plasma glucose (FPG) was assessed using an enzymatic (glucose oxidase-peroxide) in vitro test (Autoanalyzer; Echo Plus Corp., Rome, Italy). Fasting serum insulin concentration was also assessed using ELISA kits (Diametra Corp., Milan, Italy) with a sensitivity of $2 \mathrm{IU} / \mathrm{mL}$. The concentrations of serum triglyceride (TG), serum cholesterol, high-density lipoprotein (HDL) cholesterol, and low-density lipoprotein (LDL) cholesterol were measured by enzymatic colorimetric techniques (Pars Test Iran kit, with a sensitivity of $1 \mathrm{mg} / \mathrm{dL}$ for TG, serum LDL cholesterol and HDL cholesterol, and $3 \mathrm{mg} / \mathrm{dL}$ for serum cholesterol). Also, the coefficients of variation for the measurements were $1.4 \%$ for TG and cholesterol and $1.5 \%$ for LDL and HDL cholesterol. The serum CRP level was measured by enzyme immunometric assay using a commercial kit

Table 1. Demographic and baseline values of anthropometric variables

\begin{tabular}{lrrrrr}
\hline \multirow{2}{*}{ Variable } & \multicolumn{3}{c}{ Group } & \\
\cline { 2 - 5 } & Cur $(\mathrm{n}=10)$ & Pla $(\mathrm{n}=10)$ & Pla $+\operatorname{Tra}(\mathrm{n}=10)$ & \multicolumn{1}{c}{ Cur+Tra $(\mathrm{n}=10)$} \\
\hline Age $(\mathrm{yr})$ & $38.9 \pm 5.40$ & $40.80 \pm 3.55$ & $38.20 \pm 5.67$ & $35.80 \pm 3.22$ & 0.128 \\
Height $(\mathrm{cm})$ & $159.09 \pm 4.64$ & $161.83 \pm 5.36$ & $159.58 \pm 6.98$ & $162.44 \pm 4.36$ & 0.441 \\
Weight $(\mathrm{kg})$ & $68.31 \pm 6.54$ & $71.31 \pm 5.73$ & $70.37 \pm 9.23$ & $74.45 \pm 6.63$ & 0.298 \\
BMl $\left(\mathrm{kg} / \mathrm{m}^{2}\right)$ & $26.96 \pm 1.90$ & $27.21 \pm 1.43$ & $27.52 \pm 1.70$ & $28.18 \pm 1.70$ & 0.425 \\
WC $(\mathrm{cm})$ & $86.40 \pm 4.94$ & $83.50 \pm 3.53$ & $90.20 \pm 4.94$ & $96.60 \pm 11.18^{* . \dagger}$ & 0.001 \\
HC $(\mathrm{cm})$ & $103.85 \pm 6.63$ & $106.55 \pm 4.59$ & $103.00 \pm 6.42$ & $108.80 \pm 6.19$ & 0.142 \\
WHR & $0.83 \pm 0.05$ & $0.78 \pm 0.02$ & $0.87 \pm 0.04^{\dagger}$ & $0.88 \pm 0.07^{\dagger}$ & $<0.001$ \\
\hline
\end{tabular}

Values are presented as mean \pm standard deviation. Data analysis was done by analysis of variance and least significant difference post-hoc tests.

${ }^{*} P<0.05$ vs. Cur group; ${ }^{\dagger} P<0.05$ vs. Pla group.

Cur, curcumin supplement; Pla, placebo; Pla+Tra, placebo+aerobic training; Cur+Tra, curcumin supplement+aerobic training; BMl, body mass index; WC, waist circumference; HC, hip circumference; WHR, waist-to-hip ratio. 
(Diagnostic Biochem Canada Co., Ontario, Canada). The homeostatic model assessment for insulin resistance (HOMA-IR) and quantitative insulin sensitivity check index (QUICKI) were calculated by the following formulas:

HOMA-IR = fasting insulin $(\mu \mathrm{U} / \mathrm{mL}) \times$ fasting blood glucose $(\mathrm{mmol} / \mathrm{L}) / 22.5$ and QUICKI $=1 /(\log [$ insulin, $\mathrm{mIU} / \mathrm{L}]+\log$ [glucose, $\mathrm{mg} / \mathrm{dL}]$ ).

\section{Statistical analysis}

The statistical analyses were performed using IBM SPSS version 19.0 (IBM Corp., Armonk, NY, USA). All data are presented as mean \pm standard deviation. The Kolmogorov-Smirnov test was used to check the normal distributions of continuous variables. Be-

Table 2. Anthropometric variables across the study period categorized by group

\begin{tabular}{|c|c|c|c|c|c|}
\hline Variable & Cur $(n=10)$ & Pla $(n=10)$ & Pla+Tra $(n=10)$ & Cur+Tra $(n=10)$ & $P^{*}$ \\
\hline Weight (kg) & & & & & 0.912 \\
\hline Before & $68.31 \pm 6.54$ & $71.31 \pm 5.73$ & $70.37 \pm 9.23$ & $74.45 \pm 6.63$ & \\
\hline After & $67.39 \pm 7.08$ & $70.47 \pm 5.98$ & $69.24 \pm 9.73$ & $73.84 \pm 6.18$ & \\
\hline$P^{\dagger}$ & 0.033 & 0.138 & 0.053 & 0.070 & \\
\hline PC & $-1.40 \pm 1.80$ & $-1.18 \pm 2.37$ & $-1.69 \pm 2.47$ & $-0.77 \pm 1.17$ & \\
\hline \multicolumn{6}{|l|}{ BMI $\left(\mathrm{kg} / \mathrm{m}^{2}\right)$} \\
\hline Before & $26.96 \pm 1.90$ & $27.21 \pm 1.43$ & $27.52 \pm 1.70$ & $28.18 \pm 1.70$ & 0.819 \\
\hline After & $26.59 \pm 2.06$ & $26.89 \pm 1.56$ & $27.05 \pm 1.85$ & $27.95 \pm 1.63$ & \\
\hline$P^{\dagger}$ & 0.035 & 0.148 & 0.056 & 0.073 & \\
\hline PC & $-1.40 \pm 1.80$ & $-1.18 \pm 2.37$ & $-1.69 \pm 2.47$ & $-0.77 \pm 1.17$ & \\
\hline WC (cm) & & & & & 0.244 \\
\hline Before & $86.40 \pm 4.94$ & $83.50 \pm 3.53$ & $90.20 \pm 4.94$ & $96.60 \pm 11.18$ & \\
\hline After & $85.35 \pm 5.56$ & $81.85 \pm 4.00$ & $84.1 \pm 6.60$ & $89.90 \pm 9.53$ & \\
\hline$P^{\dagger}$ & 0.173 & 0.045 & 0.001 & 0.026 & \\
\hline $\mathrm{PC}$ & $-1.22 \pm 2.66$ & $-1.96 \pm 2.66$ & $-6.80 \pm 4.33$ & $-6.95 \pm 7.10$ & \\
\hline $\mathrm{HC}(\mathrm{cm})$ & & & & & 0.708 \\
\hline Before & $103.85 \pm 6.63$ & $106.55 \pm 4.59$ & $103.00 \pm 6.42$ & $108.80 \pm 6.19$ & \\
\hline After & $104.00 \pm 6.81$ & $105.90 \pm 4.88$ & $102.30 \pm 5.37$ & $108.40 \pm 5.60$ & \\
\hline$P^{\dagger}$ & 0.866 & 0.301 & 0.343 & 0.479 & \\
\hline PC & $0.16 \pm 2.73$ & $-0.60 \pm 1.78$ & $-0.60 \pm 2.15$ & $-0.32 \pm 1.65$ & \\
\hline WHR & & & & & 0.458 \\
\hline Before & $0.83 \pm 0.05$ & $0.78 \pm 0.02$ & $0.87 \pm 0.04$ & $0.88 \pm 0.07$ & \\
\hline After & $0.82 \pm 0.06$ & $0.77 \pm 0.01$ & $0.82 \pm 0.06$ & $0.82 \pm 0.06$ & \\
\hline$P^{\dagger}$ & 0.340 & 0.118 & 0.002 & 0.035 & \\
\hline PC & $-1.31 \pm 4.06$ & $-1.35 \pm 2.53$ & $-6.20 \pm 4.55$ & $-6.21 \pm 7.79$ & \\
\hline
\end{tabular}

Values are presented as mean \pm standard deviation.

*Data analysis was done by analysis of covariance and least significant difference post-hoc test after adjustment for baseline values; 'Statistical analysis was done by paired sample t-test.

Cur, curcumin supplement; Pla, placebo; Pla+Tra, placebo+aerobic training; Cur+Tra, curcumin supplement+aerobic training; $\mathrm{PC}$, percent change; $\mathrm{BMI}$, body mass index; WC, waist circumference; $\mathrm{HC}$, hip circumference; WHR, waist-to-hip ratio. tween-group comparisons were performed using the analysis of covariance followed by the least significant difference post-hoc test (Bonferroni test). Within-group comparisons were performed using paired sample t-tests. A $P$-value less than 0.05 was considered statistically significant.

\section{RESULTS}

All 40 women in the study performed the intervention for 8 weeks. No one reported any adverse side effects of curcumin ingestion.

\section{Baseline characteristics of the participants in different groups of the present study}

The baseline characteristics of the participants are presented in Table 1. Regarding age, height, BMI, and HC, there were no signifi-

Table 3. Glycemic variables across the study period categorized by group

\begin{tabular}{lccccc}
\hline Variable & Cur $(\mathrm{n}=10)$ & Pla $(\mathrm{n}=10)$ & Pla $+\operatorname{Tra}(\mathrm{n}=10)$ & Cur+Tra $(\mathrm{n}=10)$ & $P^{*}$ \\
\hline Insulin $(\mu \mathrm{U} / \mathrm{mL})$ & & & & & 0.353 \\
Before & $8.07 \pm 3.79$ & $7.25 \pm 3.12$ & $7.85 \pm 2.85$ & $9.17 \pm 3.32$ & \\
After & $7.83 \pm 2.99$ & $8.38 \pm 3.64$ & $7.92 \pm 2.15$ & $7.27 \pm 2.29$ & \\
$P^{\dagger}$ & 0.844 & 0.181 & 0.942 & 0.039 & \\
PC & $10.76 \pm 60.05$ & $19.10 \pm 33.73$ & $8.09 \pm 38.02$ & $-17.43 \pm 21.73$ & \\
FPG (mg/dL) & & & & & 0.388 \\
Before & $96.50 \pm 12.05$ & $93.40 \pm 9.96$ & $92.20 \pm 6.32$ & $93.30 \pm 5.83$ & \\
After & $88.20 \pm 4.68$ & $93.40 \pm 17.57$ & $88.80 \pm 3.82$ & $90.5 \pm 7.50$ & \\
$P^{\dagger}$ & 0.041 & 1 & 0.108 & 0.389 & \\
PC & $-7.57 \pm 10.37$ & $-0.48 \pm 11.19$ & $-3.38 \pm 6.33$ & $-2.59 \pm 11.12$ & \\
HOMA-IR & & & & & 0.289 \\
Before & $2.00 \pm 1.22$ & $1.72 \pm 0.91$ & $1.78 \pm 0.64$ & $2.12 \pm 0.82$ & \\
After & $1.69 \pm 0.65$ & $1.98 \pm 1.14$ & $1.73 \pm 0.44$ & $1.61 \pm 0.50$ & \\
$P^{\dagger}$ & 0.414 & 0.245 & 0.800 & 0.053 & \\
PC & $4.84 \pm 64.27$ & $18.00 \pm 33.58$ & $4.19 \pm 36.03$ & $-18.72 \pm 25.34$ & \\
QUICKI & & & & & 0.452 \\
Before & $0.35 \pm 0.03$ & $0.36 \pm 0.02$ & $0.35 \pm 0.17$ & $0.34 \pm 0.17$ & \\
After & $0.36 \pm 0.02$ & $0.35 \pm 0.02$ & $0.35 \pm 0.01$ & $0.36 \pm 0.02$ & \\
$P^{\dagger}$ & 0.673 & 0.258 & 0.988 & 0.044 & \\
PC & $1.58 \pm 8.10$ & $-1.72 \pm 5.03$ & $0.12 \pm 4.58$ & $4.10 \pm 5.52$ & \\
\hline
\end{tabular}

Values are presented as mean \pm standard deviation.

*Data analysis was done by analysis of covariance and least significant difference post-hoc tests after adjustment for baseline values; 'Statistical analysis was done by paired sample t-test.

Cur, curcumin supplement; Pla, placebo; Pla+Tra, placebo+aerobic training; Cur+Tra, curcumin supplement+aerobic training; $\mathrm{PC}$, percent change; FPG, fasting plasma glucose; HOMA-IR, homeostatic model assessment for insulin resistance; OUICKI, quantitative insulin sensitivity check index. 
cant differences in the baselines between groups. The baseline WC in the Cur+Tra group was significantly higher than those in the Cur and placebo groups. Also, the baseline WHRs in the Cur+Tra and Pla+Tra groups were significantly higher than that of the placebo group (Table 1).

\section{The effects of aerobic training, curcumin, and their} interaction on anthropometric variables

As shown in Table 2, no significant differences were observed between groups regarding weight and BMI. However, weight and
BMI were significantly reduced (1.4\%) in the Cur group versus the baseline measurements. Regarding WC, no significant difference was observed between groups. The post-test WCs in the Pla, $\mathrm{Pla}+\mathrm{Tra}$, and Cur+Tra groups were reduced significantly compared to the baselines by $2.22 \%, 6.8 \%$, and $6.95 \%$, respectively. HC was not significantly changed between or within groups. Regarding WHR, no significant difference was observed between groups. A significant reduction of WHR was observed in the post-test Pla+ Tra and Cur+Tra groups compared to their pre-test values (Table 2).

Table 4. Lipid profiles across the study period categorized by group

\begin{tabular}{|c|c|c|c|c|c|c|}
\hline Variable & Cur $(n=10)$ & Pla $(n=10)$ & Pla+Tra $(n=10)$ & Cur+Tra $(n=10)$ & $P^{*}$ & $P^{\dagger}$ \\
\hline TC & & & & & 0.440 & 0.260 \\
\hline Before & $182.20 \pm 26.02$ & $175.20 \pm 25.71$ & $164.5 \pm 38.31$ & $188.00 \pm 33.62$ & & \\
\hline After & $174.70 \pm 26.91$ & $164.30 \pm 25.25$ & $152.80 \pm 32.17$ & $158.10 \pm 34.24$ & & \\
\hline$P$ & 0.178 & 0.120 & 0.311 & 0.088 & & \\
\hline PC & $-3.87 \pm 9.81$ & $-5.74 \pm 10.51$ & $-4.99 \pm 16.42$ & $-13.40 \pm 22.74$ & & \\
\hline TG & & & & & 0.928 & 0.819 \\
\hline Before & $107.90 \pm 39.17$ & $83.70 \pm 32.93$ & $94.00 \pm 29.34$ & $119.70 \pm 94.12$ & & \\
\hline After & $108.30 \pm 63.27$ & $83.70 \pm 22.98$ & $93.70 \pm 35.67$ & $109.5 \pm 96.19$ & & \\
\hline$P$ & 0.979 & 1.000 & 0.973 & 0.294 & & \\
\hline PC & $1.53 \pm 39.22$ & $6.77 \pm 34.23$ & $1.87 \pm 27.89$ & $-5.29 \pm 28.02$ & & \\
\hline HDL cholesterol & & & & & 0.001 & 0.001 \\
\hline Before & $53.90 \pm 10.80$ & $63.70 \pm 16.00$ & $52.50 \pm 8.18$ & $60.30 \pm 22.23$ & & \\
\hline After & $58.40 \pm 18.22$ & $54.10 \pm 16.60$ & $66.40 \pm 10.22$ & $72.60 \pm 22.92$ & & \\
\hline$P$ & 0.205 & 0.008 & 0.004 & 0.043 & & \\
\hline $\mathrm{PC}$ & $6.62 \pm 19.25^{\S}$ & $-15.04 \pm 13.89$ & $28.50 \pm 25.70^{\S}$ & $24.19 \pm 29.12^{\S}$ & & \\
\hline LDL cholesterol & & & & & 0.072 & 0.039 \\
\hline Before & $106.70 \pm 26.77$ & $94.70 \pm 24.90$ & $93.20 \pm 32.51$ & $103.60 \pm 36.56$ & & \\
\hline After & $96.70 \pm 27.64$ & $91.30 \pm 23.79$ & $73.70 \pm 28.03$ & $72.60 \pm 21.49$ & & \\
\hline$P$ & 0.151 & 0.703 & 0.020 & 0.058 & & \\
\hline PC & $8.42 \pm 20.26^{\prime \prime}$ & $-0.20 \pm 24.93^{\prime}$ & $-19.64 \pm 18.22$ & $-19.17 \pm 41.52$ & & \\
\hline TC/HDL & & & & & 0.013 & 0.023 \\
\hline Before & $3.54 \pm 1.08$ & $2.89 \pm 0.77$ & $3.18 \pm 0.77$ & $3.66 \pm 1.89$ & & \\
\hline After & $3.17 \pm 1.84$ & $3.26 \pm 0.69$ & $2.39 \pm 0.70$ & $2.77 \pm 1.53$ & & \\
\hline$P^{\ddagger}$ & 0.690 & 0.066 & 0.009 & 0.036 & & \\
\hline PC & $3.28 \pm 37.54^{1,9}$ & $14.79 \pm 17.73^{1,99}$ & $-23.27 \pm 19.58$ & $-19.65 \pm 24.48$ & & \\
\hline LDL/HDL & & & & & 0.001 & 0.001 \\
\hline Before & $2.11 \pm 0.84$ & $1.60 \pm 0.67$ & $1.81 \pm 0.66$ & $2.11 \pm 1.31$ & & \\
\hline After & $1.90 \pm 0.99$ & $1.85 \pm 0.55$ & $1.08 \pm 0.51$ & $1.27 \pm 0.88$ & & \\
\hline$P$ & 0.123 & 0.135 & 0.005 & 0.027 & & \\
\hline PC & $-12.05 \pm 18.16^{1,9}$ & $22.14 \pm 30.83^{1,9}$ & $-38.04 \pm 25.20$ & $-29.59 \pm 34.03$ & & \\
\hline
\end{tabular}

Values are presented as mean \pm standard deviation.

*Data analysis was done by analysis of covariance (ANCOVA) and least significant difference (LSD) post-hoc test after adjustment for baseline values; ${ }^{\dagger}$ Data analysis was done by ANCOVA and LSD post-hoc test after adjustment for baseline values and baseline waist circumference; ${ }^{\ddagger}$ Statistical analysis was done by paired sample t-test; ${ }^{\S} P<0.05$ vs. Pla group; "P< 0.05 vs. Cur+Tra group; ${ }^{\natural P} P<0.05$ vs. Pla+Tra group.

Cur, curcumin supplement; Pla, placebo; Pla+Tra, placebo+aerobic training; Cur+Tra, curcumin supplement+aerobic training; TC, total cholesterol; PC: percent change; TG, triglyceride; HDL, high-density lipoprotein; LDL, low-density lipoprotein; TC/HDL, TC to HDL ratio; LDL/HDL, LDL to HDL ratio. 
The effects of aerobic training, curcumin, and their interaction on glycemic variables

Glycemic variables are presented in Table 3. Regarding insulin, no significant difference was observed between groups. However, insulin was significantly reduced compared to the baseline in the Cur+Tra group. Regarding FPG, no significant difference was observed between groups. However, FPG decreased significantly by $7.5 \%$ in the Cur group compared to the baseline. HOMA-IR showed no significant changes between or within groups. However, a trend to decrease was observed in the Pla+Tra group compared to the baseline and was near statistical significance $(P=0.053)$. Regarding QUICKI , no significant difference was observed between groups. Serum QUICKI increased significantly in the Cur+Tra group compared to the baseline (Table 3).

\section{The effects of aerobic training, curcumin, and their} interaction on serum lipids and lipoproteins

Regarding the serum levels of TG and total cholesterol (TC), no significant changes were observed between or within groups (Table 4). However, there were significant differences between groups and changes within groups regarding serum HDL level. Serum HDL levels were significantly higher in the Cur, $\mathrm{Pla}+\mathrm{Tra}$, and Cur+Tra groups compared to that of the Pla group. The serum HDL level was significantly lower in the post-test than the pre-test in the Pla group. In contrast, the serum HDL level was significantly higher in posttests than pre-tests in the Pla+Tra and Cur+Tra groups (Table 4).

Regarding serum LDL level, there were significant changes between groups (Table 4). Serum LDL levels were significantly lower in the Cur and placebo groups compared to the Cur+Tra group. In addition, the post-test LDL was significantly lower in the Pla+Tra group compared to the baseline. Also, post-test LDL was on trend to decrease in the Cur+Tra group compared to the baseline $(P=$ 0.058).

TC to HDL ratio (TC/HDL) and LDL to HDL ratio (LDL/ HDL) showed the same pattern of differences (Table 4). There were significant differences between groups; the Pla+Tra and Cur+Tra groups showed lower TC/HDL and LDL/HDL compared to the Pla group. TC/HDL and LDL/HDL were lower in the Pla+Tra and Cur+Tra groups compared to the Cur group. Posttest TC/HDL and LDL/HDL in the Cur+Tra and Pla+Tra groups

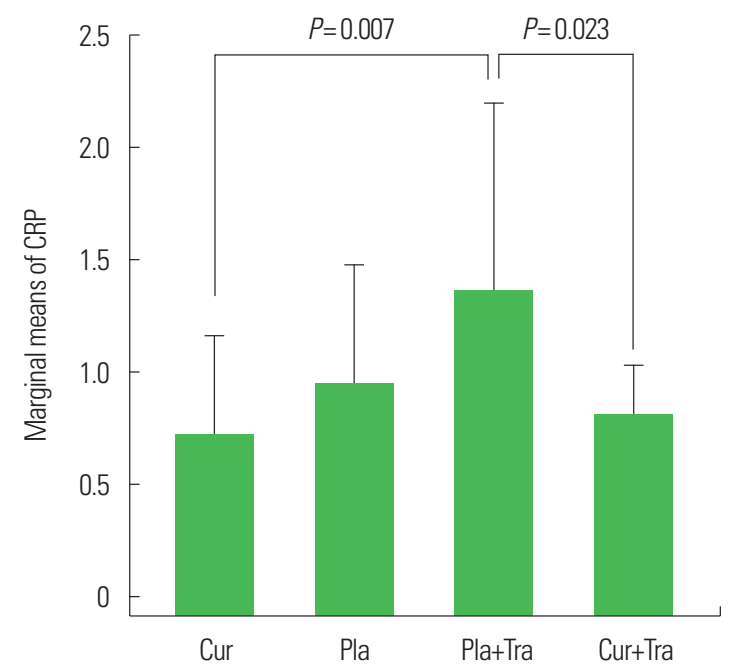

Figure 2. Marginal means of serum C-reactive protein (CRP) in each study groups after adjustment for baseline values. Between-group comparison was done by analysis of covariance. CRP, C-reactive protein; Cur, curcumin supplement; Pla, placebo; Pla+Tra, placebo+aerobic training; Cur+Tra, curcumin supplement+aerobic training.

were significantly lower than their baselines (Table 4). As shown in Fig. 2, the results of covariance analysis after adjustment for baseline values showed that the estimated marginal means of serum $\mathrm{CRP}$ were significantly higher in the Pla+Tra group than in the Cur and Cur+Tra groups. No within-group change was found for CRP compared to baseline values.

\section{DISCUSSION}

This study was conducted to investigate the effects of curcumin supplementation alone and in combination with aerobic training on body composition, CRP, glycemic variables, and lipid profiles in overweight women. The results of the present study indicated that $500 \mathrm{mg}$ per day of curcumin for 8 weeks significantly decreased weight, BMI, and FPG in overweight women. Curcumin intake with aerobic training significantly decreased serum insulin, TC/ HDL, and LDL/HDL, WC and WHR, and increased QUICKI and HDL indices. Aerobic training, with curcumin or Pla, had a positive effect on $\mathrm{WC}$ and WHR. Curcumin supplementation alone did not have any effect on WC, HC, WHR, serum insulin, HOMAIR, or QUICKI, but this study showed that curcumin supplementation had an effect on HDL compared with the Pla group and on LDL compared with the Cur+Tra group. It seems that the only ef- 
fective factor in reducing WC, WHR, serum insulin, and QUICKI was aerobic training. However, there were no significant changes between these four groups in weight, BMI, or glycemic variables before the intervention or after it. Consistent with our results, Rahimi et al. ${ }^{32}$ also reported that nano-curcumin $(80 \mathrm{mg} /$ day $)$ treatment for 3 months in 70 subjects with type 2 diabetes mellitus significantly reduced fasting blood glucose. In some previous studies, improvements in glycemic variables have been reported. ${ }^{33}$ The possible mechanism of this effect may be due to curcumin's role in insulin secretion by activating phosphoinositide 3 kinase and insulin signaling improvement. ${ }^{34}$ Also, aerobic training increases insulin's ability to stimulate muscle glucose uptake. ${ }^{35}$

In the Panahi et al. ${ }^{36}$ study that used $1,000 \mathrm{mg}$ curcumin supplementation over 3 months, weight and BMI in diabetic patients decreased significantly, similar to the results of this study. In 2018, several dose-response meta-analyses have shown the lowering effect of 8 weeks or more of curcumin supplementation on the WC variable in overweight subjects. ${ }^{13}$ The mechanism of the curcumin effect on the anthropometric indices is unclear. However, curcumin down-regulates the kinase enzyme, which has been suggested to have an important role in obesity pathogenesis. ${ }^{37} \mathrm{~A}$ high dose of curcumin also reduces obesity by the inhibition of adipocyte differentiation through suppression of the transcription factor peroxisome proliferator-activated receptor-c. ${ }^{38}$

Our results showed that the FPG was decreased significantly in the Cur group at the end of the study. Also, curcumin supplementation with aerobic training for 8 weeks reduced serum insulin level significantly and improved the QUICKI index. Various studies have shown the separate effects of curcumin and aerobic training on lipid profiles. ${ }^{39}$ Our results showed that aerobic training decreased the LDL level. The anti-hyperlipidemia effect of aerobic training is a possible reason. It can be stated that aerobic training leads to an increase in lipoprotein lipase activity. As a result, the catabolism of cholesterol-rich lipoprotein increases, and thus the amount of LDL is reduced. ${ }^{40}$ The results of the between-group analyses showed a beneficial effect of curcumin supplementation with aerobic training on HDL cholesterol levels. This finding is consistent with the study by Panahi et al. ${ }^{14}$ It is clear that Apo-A1 mediates the cholesterol transfer from cells to HDL particles, and curcumin can induce Apo-Al expression. ${ }^{41}$ Also, curcumin was found to modulate the removal of HDL by hepatocytes. ${ }^{42}$

The other interesting finding of the present study was the lower serum CRP levels in the Cur and the Cur+Tra groups compared to the Pla+Tra group. Although CRP was not statistically significant in this study, its difference in the Tra group (23.86\%) indicated that exercise was physiologically very effective. According to the previous findings, the influence of physical activity on inflammation is complex. Inflammatory markers can be affected by exercise type, intensity, duration, and consistency of the exercise sessions. ${ }^{43}$ As shown in Fig. 1, daily curcumin supplements in those who engaged in aerobic training moderated circulatory CRP. It has been shown that $\mathrm{CRP}$ production is controlled by the nuclear factor kappa $\mathrm{B}$ (NF-kB) transcription signaling pathway. A study by Shishodia et al. ${ }^{44}$ has suggested that curcumin is a well-known suppressor of the NF-kB pathway, as dietary composition is one of the considerable factors involved in the development of inflammatory-related diseases. ${ }^{45}$ Therefore, the anti-inflammatory properties of curcumin might provide a therapeutic window.

The limitations of our study were as follows. First, this was a short-term trial, and it is unknown if longer durations of supplementation could cause further improvements. Second, the impact of different dose levels was not investigated in the current research. In addition, recent studies have shown that the bioavailability of curcumin is low because of its poor absorption and rapid metabolism. Consequently, any dose-response relationship for the metabolic effects of curcumin remains unknown. Finally, our study did not consider dietary changes during the trial to monitor and normalize the dietary intakes between the experimental and control groups. The strengths of our study were the successful blinding of subjects (only two subjects claimed to know whether they were on curcumin or not), and the high rate of compliance (all subjects reported that they had taken capsules on all days).

\section{CONFLICTS OF INTEREST}

The authors declare no conflict of interest.

\section{ACKNOWLEDGMENTS}

The data used in this study were obtained from three theses in 
the Department of Sport Physiology, Faculty of Physical Education and Sport Sciences of Islamic Azad University, Central Tehran, Iran. The authors acknowledge the participants in the study, the staff participating in Shahid Khakbaz Comprehensive Health Service Center in the Yaft Abad area and the Department of Sport Physiology, Faculty of Physical Education and Sport Sciences of Islamic Azad University.

\section{AUTHOR CONTRIBUTIONS}

Study concept and design: SD; acquisition of data: SD; analysis and interpretation of data: $\mathrm{KN}, \mathrm{RA}$; drafting of the manuscript: SD; critical revision of the manuscript: SD, MAA, SA; statistical analysis: SD, KN, RA; administrative, technical, or material support: SD, KN, RA, SM; and study supervision: SD.

\section{REFERENCES}

1. Biswas T, Uddin MJ, Mamun AA, Pervin S, P Garnett S. Increasing prevalence of overweight and obesity in Bangladeshi women of reproductive age: findings from 2004 to 2014. PLoS One 2017;12:e0181080.

2. Amugsi DA, Dimbuene ZT, Mberu B, Muthuri S, Ezeh AC. Prevalence and time trends in overweight and obesity among urban women: an analysis of demographic and health surveys data from 24 African countries, 1991-2014. BMJ Open 2017; 7:e017344.

3. Morigny P, Houssier M, Mouisel E, Langin D. Adipocyte lipolysis and insulin resistance. Biochimie 2016;125:259-66.

4. Hunter T, Hart R. Reproductive consequences of obesity. London: IntechOpen; 2018.

5. Taylor VH, Forhan M, Vigod SN, McIntyre RS, Morrison KM. The impact of obesity on quality of life. Best Pract Res Clin Endocrinol Metab 2013;27:139-46.

6. Hruby A, Manson JE, Qi L, Malik VS, Rimm EB, Sun Q, et al. Determinants and consequences of obesity. Am J Public Health 2016;106:1656-62.

7. O’Brien PD, Hinder LM, Callaghan BC, Feldman EL. Neurological consequences of obesity. Lancet Neurol 2017;16:465-77. 8. Bahloul G, Aghaali G. The effects of 8 weeks interval endur- ance combined training on plasma TNF- $\alpha$, IL-10, insulin resistance and lipid profile in boy adolescents. J Pract Stud Biosci Sport 2016;4:43-54.

9. Emadyan O, Toloee EM, Farhadpour S. Effect of aerobic training in the morning and evening on lipid profile, body mass index, body fat percentage, and maximum oxygen uptake in overweight females. J Mazandaran Univ Med Sci 2017;26:211-21.

10. Vahid SS, Ramin A, Fateme H. Effect of concurrent training and flaxseed supplementation on insulin indicators and body composition in overweight women. Iran J Obstet Gynaecol Infertil 2018;21:9-21.

11. Hosseinian M, Banitalebi E, Amirhosseini SE. Effect of 12 weeks of intensive interval and combined training on apolipoprotein $\mathrm{A}$ and $\mathrm{B}$, visfatin and insulin resistance in overweight middleaged women with type 2 diabetes. Horizon Med Sci 2016;22: 237-45.

12. Westerterp KR. Changes in physical activity over the lifespan: impact on body composition and sarcopenic obesity. Obes Rev 2018;19 Suppl 1:8-13.

13. Mousavi SM, Milajerdi A, Varkaneh HK, Gorjipour MM, Esmaillzadeh A. The effects of curcumin supplementation on body weight, body mass index and waist circumference: a systematic review and dose-response meta-analysis of randomized controlled trials. Crit Rev Food Sci Nutr 2020;60:171-80.

14. Panahi Y, Khalili N, Sahebi E, Namazi S, Reiner Ž, Majeed M, et al. Curcuminoids modify lipid profile in type 2 diabetes mellitus: a randomized controlled trial. Complement Ther Med 2017;33:1-5.

15. Sharma S, Kulkarni SK, Chopra K. Curcumin, the active principle of turmeric (Curcuma longa), ameliorates diabetic nephropathy in rats. Clin Exp Pharmacol Physiol 2006;33:940-5.

16. Chainani-Wu N. Safety and anti-inflammatory activity of curcumin: a component of tumeric (Curcuma longa). J Altern Complement Med 2003;9:161-8.

17.Akbari M, Lankarani KB, Tabrizi R, Ghayour-Mobarhan M, Peymani P, Ferns G, et al. The effects of curcumin on weight loss among patients with metabolic syndrome and related disorders: a systematic review and meta-analysis of randomized controlled trials. Front Pharmacol 2019;10:649.

18. Yoshizaki T, Schenk S, Imamura T, Babendure JL, Sonoda N, 
Bae EJ, et al. SIRT1 inhibits inflammatory pathways in macrophages and modulates insulin sensitivity. Am J Physiol Endocrinol Metab 2010;298:E419-28.

19. Qadir MI, Naqvi ST, Muhammad SA. Curcumin: a polyphenol with molecular targets for cancer control. Asian Pac J Cancer Prev 2016;17:2735-9.

20. Esmaily H, Sahebkar A, Iranshahi M, Ganjali S, Mohammadi A, Ferns G, et al. An investigation of the effects of curcumin on anxiety and depression in obese individuals: a randomized controlled trial. Chin J Integr Med 2015;21:332-8.

21. Shanmugam MK, Rane G, Kanchi MM, Arfuso F, Chinnathambi A, Zayed ME, et al. The multifaceted role of curcumin in cancer prevention and treatment. Molecules 2015;20:2728-69.

22. Soetikno V, Sari FR, Sukumaran V, Lakshmanan AP, Harima M, Suzuki K, et al. Curcumin decreases renal triglyceride accumulation through AMPK-SREBP signaling pathway in streptozotocin-induced type 1 diabetic rats. J Nutr Biochem 2013;24:796802.

23. Kapakos G, Youreva V, Srivastava AK. Attenuation of endothelin-1-induced PKB and ERK1/2 signaling, as well as Egr-1 expression, by curcumin in A-10 vascular smooth muscle cells. Can J Physiol Pharmacol 2012;90:1277-85.

24. Belcaro G, Cesarone MR, Dugall M, Pellegrini L, Ledda A, Grossi MG, et al. Product-evaluation registry of Meriva ${ }^{\circledR}$, a curcumin-phosphatidylcholine complex, for the complementary management of osteoarthritis. Panminerva Med 2010; 52(2 Suppl 1):55-62.

25. Meja KK, Rajendrasozhan S, Adenuga D, Biswas SK, Sundar IK, Spooner G, et al. Curcumin restores corticosteroid function in monocytes exposed to oxidants by maintaining HDAC2. Am J Respir Cell Mol Biol 2008;39:312-23.

26. Jazayeri-Tehrani SA, Rezayat SM, Mansouri S, Qorbani M, Alavian SM, Daneshi-Maskooni M, et al. Nano-curcumin improves glucose indices, lipids, inflammation, and Nesfatin in overweight and obese patients with non-alcoholic fatty liver disease (NAFLD): a double-blind randomized placebo-controlled clinical trial. Nutr Metab (Lond) 2019;16:8.

27. Alizadeh Z, Halabchi F, Noormohammadpour P, Mazaheri R, Tabesh MR. The effect of one session of dynamic high intensity exercise on calorie intake and appetite indices in overweight and obese sedentary women. Pajoohande 2016;21:238-46.

28. Pate RR, Pratt M, Blair SN, Haskell WL, Macera CA, Bouchard C, et al. Physical activity and public health: a recommendation from the Centers for Disease Control and Prevention and the American College of Sports Medicine. JAMA 1995;273: 402-7.

29. Kuptniratsaikul V, Dajpratham P, Taechaarpornkul W, Buntragulpoontawee M, Lukkanapichonchut P, Chootip C, et al. Efficacy and safety of Curcuma domestica extracts compared with ibuprofen in patients with knee osteoarthritis: a multicenter study. Clin Interv Aging 2014;9:451-8.

30. Tanaka H, Monahan KD, Seals DR. Age-predicted maximal heart rate revisited. J Am Coll Cardiol 2001;37:153-6.

31. Boyle C, Dobson A, Egger G, Bennett SA. Waist-to-hip ratios in Australia: a different picture of obesity. Aust J Nutr Diet 1993;50:57-64.

32. Rahimi HR, Mohammadpour AH, Dastani M, Jaafari MR, Abnous K, Ghayour Mobarhan M, et al. The effect of nanocurcumin on $\mathrm{HbAlc}$, fasting blood glucose, and lipid profile in diabetic subjects: a randomized clinical trial. Avicenna J Phytomed 2016;6:567-77.

33. Na LX, Li Y, Pan HZ, Zhou XL, Sun DJ, Meng M, et al. Curcuminoids exert glucose-lowering effect in type 2 diabetes by decreasing serum free fatty acids: a double-blind, placebo-controlled trial. Mol Nutr Food Res 2013;57:1569-77.

34. Kim Y, Clifton P. Curcumin, cardiometabolic health and dementia. Int J Environ Res Public Health 2018;15:2093.

35. Hall KE, McDonald MW, Grisé KN, Campos OA, Noble EG, Melling CW. The role of resistance and aerobic exercise training on insulin sensitivity measures in STZ-induced type 1 diabetic rodents. Metabolism 2013;62:1485-94.

36. Panahi Y, Khalili N, Sahebi E, Namazi S, Karimian MS, Majeed $\mathrm{M}$, et al. Antioxidant effects of curcuminoids in patients with type 2 diabetes mellitus: a randomized controlled trial. Inflammopharmacology 2017;25:25-31.

37. Wang SL, Li Y, Wen Y, Chen YF, Na LX, Li ST, et al. Curcumin, a potential inhibitor of up-regulation of TNF-alpha and IL-6 induced by palmitate in 3T3-L1 adipocytes through NFkappaB and JNK pathway. Biomed Environ Sci 2009;22:32-9. 38. Lee YK, Lee WS, Hwang JT, Kwon DY, Surh YJ, Park OJ. 
Curcumin exerts antidifferentiation effect through AMPKalpha-PPAR-gamma in 3T3-L1 adipocytes and antiproliferatory effect through AMPKalpha-COX-2 in cancer cells. J Agric Food Chem 2009;57:305-10.

39. Yang YS, Su YF, Yang HW, Lee YH, Chou JI, Ueng KC. Lipidlowering effects of curcumin in patients with metabolic syndrome: a randomized, double-blind, placebo-controlled trial. Phytother Res 2014;28:1770-7.

40. Guo W, Kawano H, Piao L, Itoh N, Node K, Sato T. Effects of aerobic exercise on lipid profiles and high molecular weight adiponectin in Japanese workers. Intern Med 2011;50:389-95.

41. Zhao JF, Ching LC, Huang YC, Chen CY, Chiang AN, Kou YR, et al. Molecular mechanism of curcumin on the suppression of cholesterol accumulation in macrophage foam cells and atherosclerosis. Mol Nutr Food Res 2012;56:691-701.

42. Panahi Y, Ahmadi Y, Teymouri M, Johnston TP, Sahebkar A.
Curcumin as a potential candidate for treating hyperlipidemia: a review of cellular and metabolic mechanisms. J Cell Physiol 2018;233:141-52.

43. Wilund KR. Is the anti-inflammatory effect of regular exercise responsible for reduced cardiovascular disease? Clin Sci (Lond) 2007;112:543-55.

44. Shishodia S, Amin HM, Lai R, Aggarwal BB. Curcumin (diferuloylmethane) inhibits constitutive NF-kappaB activation, in duces G1/S arrest, suppresses proliferation, and induces apoptosis in mantle cell lymphoma. Biochem Pharmacol 2005;70: 700-13.

45. Namazi N, Larijani B, Azadbakht L. Dietary inflammatory index and its association with the risk of cardiovascular diseases, metabolic syndrome, and mortality: a systematic review and meta-analysis. Horm Metab Res 2018;50:345-58. 